\title{
The Critical Need for Pharmacist Involvement in the Management of Patients With Hepatitis C
}

\author{
Linda M. Spooner, PharmD, BCPS
}

$\mathrm{H}$ epatitis C virus (HCV) infection is a global and national health issue, affecting an estimated 170 million people worldwide and 4 million people in the United States. ${ }^{1,2}$ Approximately $80 \%$ of infected individuals will develop chronic HCV infection, which may result in substantial morbidity and mortality due to the development of hepatic fibrosis, liver cirrhosis, and hepatocellular carcinoma. ${ }^{1}$ Annually, between 8,00010,000 deaths result from HCV infection in the United States. ${ }^{2}$ It has been estimated that direct medical costs secondary to HCV infection will reach $\$ 10.7$ billion in the United States by the year 2019. ${ }^{2}$ The high cost of HCV drug therapy, close patient followup, and laboratory monitoring for virologic response and adverse effects contribute to this staggering figure.

In this issue of JMCP, Mariño et al. present the results of their study assessing the impact of pharmacists in management of patients with chronic HCV infection, specifically genotype 1 infection, in a secondary-care hospital in Catalonia, Spain. ${ }^{3}$ A convenience sample of 50 treatment-naive patients initiating therapy with once-weekly subcutaneous peginterferon alfa- $2 b$ and twice-daily oral ribavirin received consultation from pharmacists throughout the entire course of therapy. Pharmacists provided oral and written disease and drug information, including education about treatment of adverse drug reactions, proper storage of medications, methods of drug administration, and management of missed doses. These visits were provided in tandem with the attending hepatologists at the study site. The authors described an overall sustained virologic response (SVR) of $48 \%$, which is comparable to that observed in other trials of patients with genotype $1 \mathrm{HCV}^{4}$ They noted that this SVR may have been increased by the low rate of early treatment discontinuation in the study. Additionally, the overall mean adherence rate was $85.7 \%$; the adherence rate in the patients achieving SVR was an impressive $95.5 \%{ }^{3}$

The authors are commended for focusing their study on the benefits of pharmacist involvement in management of chronic $\mathrm{HCV}$ infection. They intervened on patients with genotype 1 virus, a type that is challenging to eradicate due to the lengthy treatment duration required. The study was strengthened by the visual assessment of adherence at each visit using inspection of patients' pill boxes, providing a more accurate assessment of adherence than does patient self-report. The role of the pharmacist is clearly delineated in this study, from the pharmacist's role as an educator to motivator for those patients experiencing an early virologic response at 12 weeks. Along with their face-to- face meetings with patients, the pharmacists' close participation with hepatologists facilitated a multidisciplinary collaboration that enhanced patient care. Similarly, the 24-hour pharmacist support, provided to these patients after hours and on weekends and holidays, was an invaluable addition to the care that they received.

The authors acknowledged that one of the limitations of their study was its lack of a comparison group. Without a control group, it is impossible to quantify the impact that pharmacists had on the SVR in this study. Additionally, this study included only patients with genotype $1 \mathrm{HCV}$ infection. Although this genotype is the most common in the United States (comprising $70 \%-80 \%$ of patients) ${ }^{2}$ and in Spain, patients with other genotypes would also likely benefit from pharmacist intervention. Although patients with genotypes 2 and 3 infection typically receive shorter courses of therapy ( 24 weeks) as compared with genotypes 1 and 4 (48 weeks), ${ }^{2}$ the pharmacist can still strongly influence patients with respect to education, adherence, and management of adverse effects. Lastly, this study excluded patients who were coinfected with human immunodeficiency virus (HIV) infection. Since approximately 25\%-33\% of HIVpositive individuals are coinfected with $\mathrm{HCV},{ }^{2}$ a potentially large segment of the HCV-infected population was not considered. The complicated pharmacotherapeutic regimens of coinfected subpopulations of patients underscore more clearly the need for pharmacist involvement.

\section{Do Pharmacists Make a Difference in Management of HCV Infection?}

A paucity of data are available to illustrate the benefits of pharmacist involvement in HCV management. In 2007, Smith et al. published a pilot study that retrospectively evaluated outcomes in a pharmacist-managed HCV clinic at the Los Angeles Department of Veterans Affairs (VA) Healthcare System. ${ }^{5}$ Overall, 17 of the 27 enrolled patients (63\%) achieved a SVR. The authors concluded that their clinic was able to achieve a SVR rate comparable to that of physician-run clinics, while also efficiently providing patient support and education. A major limitation of this study was its lack of a comparison group without pharmacist involvement. Additionally, there may have been bias in the selection of motivated patients for this pilot study, resulting in a higher SVR rate and no patients lost to follow-up. Adherence assessment was not performed. Nevertheless, the study included patients with genotype 1, as well as genotypes 
2 and $3 \mathrm{HCV}$, and provided information about detailed and broad-ranging responsibilities for the pharmacist working in the HCV clinic setting.

The only other literature published about the role of the pharmacist in $\mathrm{HCV}$ management is descriptive in nature. ${ }^{6}$ Kolor described a pharmacist-managed HCV clinic at the VA Long Beach Healthcare System, where specially trained pharmacists provided extensive initial and follow-up visits. During the visits, pharmacists independently adjusted doses of drug therapy, ordered laboratory tests to assess virologic response and adverse effects, and referred patients to subspecialists as needed. This paper did not describe SVR rates or adverse event rates observed in patients seen in the clinic by pharmacists.

Rodis and Kibbe described an HCV support group developed by a pharmacist and a nurse practitioner in Central Ohio. ${ }^{7}$ Although no data were gathered with respect to virologic outcomes, adherence, or adverse effects, the authors noted that they received an increased number of patient referrals to their ambulatory care clinic as a result of their involvement with the support group.

Although preliminary and lacking in methodological rigor, these descriptions suggest the potential for pharmacists to play an important part in the management of HCV infection. Pharmacists are in an ideal position to provide care for patients with $\mathrm{HCV}$ due to the long duration of therapy, need for close monitoring of adverse effects and laboratory values, and potential dose adjustments required. ${ }^{6}$ It is a natural role for pharmacists to fill, similar to the function that pharmacists have in the management of other chronic disease states, such as diabetes mellitus, hyperlipidemia, and asthma. ${ }^{8}$ Quantifying the benefits of pharmacists in the management of patients with HCV infection will likely permit greater support for their involvement. This important topic area warrants future study using a comparative design in order to assess the costs and benefits of pharmacist involvement.

\section{Why Aren't More Pharmacists Involved in HCV Clinics?}

While recognizing the shortage of data quantifying the benefits of pharmacist participation in the management of individuals with chronic HCV infection, one wonders why more pharmacists are not becoming involved in these clinics. Unfortunately, most ambulatory care pharmacists have difficulty allocating time to spend in an additional clinic each week. There may be an overwhelming need for pharmacist management of more prevalent chronic issues such as diabetes mellitus, asthma, and anticoagulation that precludes the ambulatory care pharmacist from diversifying into the HCV arena. Additionally, conflicts between the HCV clinic times and administrative meetings and responsibilities prevent the university-based clinical pharmacist from consistently being involved in the clinic.

Other issues that arise include monetary considerations and awareness of opportunities. Many HCV clinics cannot afford to hire a clinical pharmacist to participate in patient care. Clinic physicians are often unaware of pharmacists in the area who would be interested in developing a segment of their practice in HCV management. Further, clinical pharmacists may not have considered becoming involved in this area. Ambulatory care pharmacists often assume that clinical pharmacists specializing in infectious diseases will cover HCV clinic management, and vice versa. Many pharmacists are unaware of which practitioners their patients are seeing for management of their HCV infection, since so many different specialists may provide this care, including gastroenterologists, infectious diseases specialists, HIV subspecialists, and primary care physicians. Although no formal certification or training is required for pharmacists to care for patients with HCV, clinical pharmacists often feel a level of intimidation about the complexity of $\mathrm{HCV}$ treatment with respect to medication dosing and adverse effect management. All of these issues contribute to missed opportunities for optimal management of patients with $\mathrm{HCV}$ infection.

A multidisciplinary approach to the management of this disease will permit further research into the beneficial effects that pharmacists could potentially have on treatment success as well as discovery of avenues for funding. Perhaps an increased awareness of the need for pharmacist participation in HCV clinics can be accomplished with inclusion of pharmacy practice residents in the HCV clinic setting, thereby fostering an early interest in management of this disease. Additionally, pharmacists who manage HIV-positive individuals in the clinic setting are also in an ideal position to become involved in treatment of HCV infection as well, through collaboration with other pharmacists who focus their practices in the ambulatory care arena.

\section{Author}

LINDA M. SPOONER, PharmD, BCPS, is Associate Professor of Pharmacy Practice, Massachusetts College of Pharmacy and Health Sciences, School of Pharmacy-Worcester/Manchester, Worcester, Massachusetts.

AUTHOR CORRESPONDENCE: Linda M. Spooner, PharmD, BCPS, Massachusetts College of Pharmacy and Health Sciences, 19 Foster Street, Worcester, MA 01608. Tel.: 508.373.5696; E-mail: linda.spooner@mcphs.edu 


\section{The Critical Need for Pharmacist Involvement in the Management of Patients With Hepatitis C}

\section{DISCLOSURES}

The author has served as a consultant to Vertex Pharmaceuticals, Inc.

\section{REFERENCES}

1. World Health Organization. Hepatitis C. Fact sheet No. 164, revised October 2000. Available at: http://www.who.int/mediacentre/factsheets/ fs164/en/. Accessed February 5, 2009

2. Dienstag JL, McHutchison JG. American Gastroenterological Association technical review on the management of hepatitis C. Gastroenterology. 2006;130:231-64.

3. Mariño EL, Alvarez-Rubio L, Miró S, et al. Pharmacist intervention in treatment of patients with genotype 1 chronic hepatitis C. J Manag Care Pharm. 2009;15(2):147-50
4. Manns MP, McHutchison JG, Gordon SC, et al. Peginterferon alfa$2 \mathrm{~b}$ plus ribavirin compared with interferon alfa- $2 \mathrm{~b}$ plus ribavirin for initial treatment of chronic hepatitis $\mathrm{C}$ : a randomised trial. Lancet. 2001;358(9286):958-65.

5. Smith JP, Dong MH, Kaunitz JD. Evaluation of a pharmacist-managed hepatitis C care clinic. Am J Health Syst Pharm. 2007;64(6):632-36.

6. Kolor B. Patient education and treatment strategies implemented at a pharmacist-managed hepatitis $C$ virus clinic. Pharmacotherapy. 2005;25(9):1230-41.

7. Rodis JL, Kibbe P. Development of a hepatitis C support group. Am J Health Syst Pharm. 2006;63(17):1594-96.

8. Snella KA, Sachdev GP. A primer for developing pharmacist-managed clinics in the outpatient setting. Pharmacotherapy. 2005;23(9):1153-66. 